\title{
Luminous hot accretion flows: thermal equilibrium curve and thermal stability
}

\author{
Feng Yuan \\ Harvard-Smithsonian Center for Astrophysics, 60 Garden Street, Cambridge, MA 02138 \\ fyuan@cfa.harvard.edu
}

\begin{abstract}
In a previous paper, we presented the global solution of a new accretion flow model, namely luminous hot accretion flows (LHAFs). In this Letter, we first show the corresponding thermal equilibrium curve of LHAFs in the mass accretion rate vs. surface density diagram. Then we examine its thermal stability again local perturbations. We find that LHAFs are thermally unstable when thermal conduction is neglected. However, when the accretion rate is not very large, the timescale of the growth of perturbations is longer than the accretion timescale, therefore the instability has no dynamical effect on the accretion flow. When the accretion rate is large, the perturbations can grow very fast at a certain radius. As a result, some cold clumps may form and the accretion flow will become multi-phase.
\end{abstract}

Subject headings: accretion, accretion disks - black hole physics - galaxies: active galaxies: nuclei - hydrodynamics

\section{Introduction: the physics of the new hot accretion disk solution}

There has been a great interest to the accretion process around black holes since this process is very common in a variety of astrophysical systems. The most famous accretion solution is the standard thin disk model (hereafter SSD) developed by Shakura \& Sunyaev (1973) and others (see Frank et al. 1992 for a review). The accretion gas forms a geometrically thin, optically thick disk. The temperature of the gas is in the range $10^{5}-10^{7} \mathrm{~K}$, depending on the black hole mass and the accretion rate.

The second solution was discovered by Shapiro, Lightman \& Eardley (1976; hereafter SLE). Compared to the standard thin disk, the gas is optically thin and its temperature is much higher, $T_{e} \sim 10^{9} \mathrm{~K}$, so SLE is potentially able to explain the X-ray emission observed in 
X-ray binaries and active galactic nuclei. Pringle (1976) found that SLE solution is thermally unstable although it is not clear what is the consequence of such instability.

In both solutions mentioned above, the energy advection is neglected. In other words, all of the viscously dissipated energy was assumed to be radiated away immediately. When the mass accretion rate is higher than the Eddington rate $\dot{M}_{\text {Edd }}\left(\equiv 10 L_{\text {Edd }} / c^{2}\right)$, it was found, however, that the large optical depth traps most of the photons therefore most of the viscously dissipated energy is stored in the gas and advected into the black hole rather than radiated away ("slim disk"; Abramowicz et al. 1988; see also Begelman \& Meier 1982).

The SLE solution was further developed by taking into account the energy advection which plays a dominant role in the energy balance of ions (advection-dominated accretion flow-ADAF; Ichimaru 1977; Rees et al. 1982; Narayan \& Yi 1994, 1995; Abramowicz et al. 1995; see reviews by Narayan, Mahadevan \& Quataert 1998; Kato, Fukue \& Mineshige 1998). The energy advection is included in the ions energy equation, $Q_{\mathrm{adv}}=Q_{\mathrm{vis}}-Q_{\mathrm{ie}}$. Here $Q_{\mathrm{adv}}, Q_{\mathrm{vis}}$ and $Q_{\mathrm{ie}}$ are the rates of energy advection, viscous heating and Coulomb cooling per unit area of the accretion disk, respectively. In a typical ADAF, since the density of gas is very low, the Coulomb cooling is very inefficient, so $Q_{\mathrm{ie}} \ll Q_{\mathrm{vis}} \approx Q_{\mathrm{adv}}$, i.e., the viscous heating is balanced by advection cooling. Since $Q_{\text {ie }} \propto \dot{M}^{2}$ and $Q_{\text {vis }} \propto \dot{M}$, there exists a critical rate $\dot{M}_{1}$, which is determined by $Q_{\text {vis }} \approx Q_{\text {ie }}$. At this rate, a large fraction of the viscously dissipated energy is transferred to the electrons and radiated away, so the accretion flow ceases to be advection-dominated. For the "standard" radiative cooling processes of electrons, namely synchrotron, bremsstrahlung emission and their Comptonization, we roughly have $\dot{M}_{1} \sim$ $\alpha^{2} \dot{M}_{\text {Edd }}{ }^{1}$ Above $\dot{M}_{1}$, it was thought previously that no hot solution exists and only the standard thin disk solution is viable.

However, our recent work (Yuan 2001, hereafter Paper I) indicated that this is not true: above $\dot{M}_{1}$, a new hot accretion solution exists. To illustrate, let's write out the formula of the energy advection of ions,

$$
Q_{\mathrm{adv}}=\rho H v_{r}\left[\frac{k}{\mu m_{\mu}} \frac{1}{\gamma-1} \frac{d T}{d r}-\frac{k T}{\mu m_{\mu}} \frac{1}{\rho} \frac{d \rho}{d r}\right] \equiv Q_{\mathrm{int}}-Q_{\mathrm{com}}
$$

i.e., the energy advection consists of two terms, namely the "internal energy gradient' term $Q_{\text {int }}$ and the compression work $Q_{\text {com. }}$. When $\dot{M}>\dot{M}_{1}, Q_{\text {vis }}-Q_{i e}<0$, but there obviously exists another critical accretion rate, $\dot{M}_{2}$, determined by $Q_{\text {com }}+Q_{\text {vis }} \approx Q_{i e}$. Below $\dot{M}_{2}$, we still have $Q_{\text {int }}>0$. Therefore, the accretion flow can remain hot if it starts out hot. We denote

\footnotetext{
${ }^{1}$ When additional cooling, such as the Comptonization of external soft photons, is included, the value of $\dot{M}_{1}$ will decrease (Esin, McClintock \& Narayan 1997). See Zdziarski (1998) for a discussion of ADAF with a more general electrons energy equation.
} 
this new branch of accretion solution as luminous hot accretion flow (LHAF hereafter). Paper I presents the full analysis of the dynamics of LHAF. Compared to ADAFs, $T_{e}$ in LHAFs is moderately lower while the optical depth is higher. The detailed calculation indicates that depending on the value of $\dot{M}$, there are two types of LHAF solution. When $\dot{M}_{1} \lesssim \dot{M} \lesssim \dot{M}_{2}$, with $\dot{M}_{2}$ can be as high as $5 \dot{M}_{1}$, the accretion gas in LHAF is hot throughout the disk; when

$\dot{M} \gtrsim \dot{M}_{2}$, up to $\dot{M}_{\text {Edd }}$, within a certain radius the density of the accretion gas is so high that even the sum of compression work and viscous dissipation can not balance the Coulomb cooling. As a result, the hot accretion flow will collapse onto the equatorial plane and form an optically thick cold annulus.

LHAFs are along the line of ADAFs - the equations describing them are completely the same and we just extend ADAFs to higher accretion rates. However, the dynamics of an LHAF is quite different with an ADAF. In an ADAF, the energy advection term is positive, i.e., the advection plays a "cooling" role from the Lagrangian point of view. But in an LHAF, the advection term is negative, so it plays a "heating" role, or in the language of entropy, it is the conversion of entropy together with viscous dissipation that supplies the radiation of LHAFs. In this sense, an LHAF is dynamically similar with Bondi accretion and cooling flow in galactic clusters.

In this context we can understand why previous authors didn't find this branch of solution. In Narayan \& Yi (1995) and Esin et al. (1997), they a priori set the advection factor " $f$ " ( $\left.\equiv Q_{\text {adv }} / Q_{\text {vis }}\right)$ as positive, so they only obtain the ADAF solution. This is also the reason why Abramowicz et al. (1995) and Chen et al. (1995) didn't find this solution in their local analysis because they set their parameter $\xi$ (see eq. (6) of the present paper) as positive.

In this Letter, we will first show the thermal equilibria of LHAFs in the $\dot{M}$ vs. $\Sigma$ (surface density) diagram (§2). Such a diagram is widely used in the study of accretion disks (e.g., Luo \& Liang 1994; Abramowicz et al. 1995; Chen et al. 1995; Kusunose \& Mineshige 1996; Bjönsson et al. 1996). Then in $\S 3$ we investigate the thermal stability of LHAFs against local perturbations.

\section{Thermal equilibrium curve of LHAFs}

For the purpose of comparison, we adopt almost exactly the same equations as Chen et al. (1995; see also Abramowicz et al. 1995). We take a one-temperature accretion flow as an example for the purpose of simplicity and comparison with previous work. The equations are, 


$$
\begin{gathered}
\dot{M}=-2 \pi R \Sigma v_{r}, \\
\nu \Sigma=\frac{\dot{M}}{3 \pi} f_{*} g^{-1}, \\
Q_{\mathrm{adv}}=Q_{\mathrm{vis}}-Q_{\mathrm{rad}} .
\end{gathered}
$$

They represent conservations of mass, angular momentum, and energy, respectively. Here $\nu=\frac{2}{3} \alpha c_{s} H$ is the kinetic viscosity coefficient, $\Sigma=2 \rho H$ is the surface density, $f_{*}=1-$ $9 \Omega\left(3 R_{s}\right) /\left[\Omega(R)\left(R / R_{s}\right)^{2}\right]$ with $R_{s}=2 G M / c^{2}, g=-\frac{2}{3}(d \ln \Omega / d \ln R), Q_{\mathrm{vis}}, Q_{\mathrm{adv}}, Q_{\mathrm{rad}}$ are the rates of viscous dissipation, advection, and radiation per unit area of the disk, respectively. They have the following form (Chen et al. 1995),

$$
\begin{gathered}
Q_{\mathrm{vis}}=\frac{3 \dot{M}}{4 \pi} \Omega^{2} f_{*} g, \\
Q_{\mathrm{adv}}=-\Sigma v_{r} \frac{p}{\rho} \xi=\frac{\dot{M}}{2 \pi R^{2}} \frac{p}{\rho} \xi \\
Q_{\mathrm{rad}}=8 \sigma T^{4}\left(\frac{3 \tau}{2}+\sqrt{3}+\frac{8 \sigma T^{4}}{Q_{\mathrm{brem}}}\right)^{-1} .
\end{gathered}
$$

The optical depth is the sum of scattering and absorption optical depth, $\tau=\tau_{\text {es }}+\tau_{\text {abs }}$, with $\tau_{\text {es }}=(1 / 2) k_{\text {es }} \Sigma$ and $\tau_{\text {abs }}=Q_{\text {brem }} /\left(8 \sigma T^{4}\right)$. The equation of state is $p=p_{\text {gas }}+p_{\text {rad }}$, with $p_{\text {gas }}=$ $\frac{\Re}{\mu} \rho T$, and $p_{\text {rad }}=\frac{Q_{\mathrm{rad}}}{4 c}\left(\tau+\frac{2}{\sqrt{3}}\right)$ (Abramowicz et al. 1996). The bremsstrahlung radiation is the sum of electron-ion and electron-electron bremsstrahlung, $Q_{\mathrm{brem}}=2 H\left(q_{e i}+q_{e e}\right)$. The formula for $q_{e i}$ and $q_{e e}$ are from Narayan \& Yi (1995). Other useful equations are the hydrostatic balance equation in the vertical direction of the disk, $H=c_{s} / \Omega_{\mathrm{K}}$ with $c_{s}=(p / \rho)^{1 / 2}$ is the local sound speed. The Paczyński \& Wiita (1980) potential is used to mimic the geometry of the black hole.

One noticeable parameter is $\xi$ in eq. (6). As we argued in the last section that the advection term in an LHAF is negative, therefore we should set $\xi<0$ to recover this solution. The exact value of $\xi$ should be obtained by solving the global solution of accretion flow. Adopting parameters of $\dot{m} \equiv \dot{M} / \dot{M}_{\text {Edd }}=10^{-1.6}, \alpha=0.1$, with outer boundary conditions of $R_{\text {out }}=10^{3} R_{s}, T_{\text {out }}=2 \times 10^{9} \mathrm{~K}$, and $v / c_{s}=0.4$, we obtained one exact global solution of one temperature accretion flow (LHAF) and find $\xi \approx-0.3$ at $r=5 R_{s}$. Since our purpose is only to show the equilibria of LHAFs in the $\dot{m}-\Sigma$ diagram, here we simply assume $\xi$ is independent of radius for LHAFs. As in previous work (e.g. Abramowicz et al. 1995), we set $\xi=1$ to recover all other accretion solutions. The exact value of $\xi$ doesn't affect our qualitative results. However, because of the crudeness of the value of $\xi$, from such local 
analysis we can't obtain the exact quantitative results such as the ranges of accretion rates to which various accretion disk models correspond.

Figure 1 shows the thermal equilibrium curves of different solutions. The parameters are $\alpha=0.1, R / R_{s}=5$. The thin solid lines are for ADAF, SLE, SSD and slim disk, obtained for $\xi=1$. These lines are the same as in Abramowicz et al. (1995) or Chen et al. (1995). The three "U" shaped thick lines are new. They denote LHAFs with $\xi=-1$ (solid), -0.1 (dotted) and -10 (dashed), respectively. Such lines extend from optically thin regime to optically thick regime, bridging the SLE and the SSD solutions. The squares denote the location where the optical depth is unity. It is not clear what physical implications the "bridging" has, or it might just be a mathematical trick. But we note in this context that in the optically thick slim disk solutions, they did find solutions with the advection term being negative in some cases (Abramowicz et al. 1988; Szuszkiewicz, private communication). Some segment of the "U" curves are superimposed on the SLE and SSD lines. We find that advection in these "superimposed" segment is equal to zero, same with SLE and SSD. Only the "separate" segment denotes the genuine LHAFs, since for this segment the energy advection is negative and plays a significant role in the energy balance of ions. We see that above the critical accretion rate of ADAF $\dot{m}_{1}(\sim 0.1)$, both ADAF and SLE disappear and LHAF is the only available hot solution. This is consistent with our analysis in $\S 1$.

For comparison, we also solve the global solutions of the standard accretion equations describing a one-temperature accretion flow (see, e.g., Yuan 1999 for the equations except that the radiative term is now replaced by eq. (7) of the present paper). The parameters are the same as in Fig. 1, i.e., $M=10 M_{\odot}, \alpha=0.1$. The results are denoted by the filled circles (for ADAFs) and triangles (LHAFs) in Fig. 1. We can see that our local algebraic analysis is qualitatively consistent with the global results, i.e., when the accretion rate is lower than a certain value, the solutions are ADAFs; while above this value, the solutions are LHAFs.

We would like to note that this "U" shaped line is completely different from the line with the similar shape in Chen et al. (1995), which was obtained by setting a very large viscous parameter $\alpha>\alpha_{c r}$ with $\alpha_{c r} \sim 0.2$ or larger. In fact, a later more accurate treatment of microphysics and the inner boundary condition by Björnsson et al. (1996) indicated that $\alpha_{c r}>1$, therefore, the "U" shaped branch in Chen et al. (1995) is unphysical.

\section{Thermal stability}

From the density profile of the global solution in Paper I, we know that LHAFs are viscously stable. An important problem is the thermal stability of LHAFs. Before doing 
that, we note that an LHAF is dynamically very similar to a cooling flow in galactic clusters. The angular momentum of cooling flow is generally neglected and the dominant heating mechanism is compression work. In other words, the entropy of the cooling flow is converted to radiation. Many authors have studied its thermal stability and concluded that it is unstable against local perturbations if thermal conduction is neglected (e.g., Fabian \& Nulsen 1977; Mathews \& Bregman 1978; Nulsen 1986). But when thermal conduction is included, it was found that the cooling flow is thermally stable (Zakamska \& Narayan 2003; Kim \& Narayan 2003).

Now let's investigate the thermal stability of LHAFs against local perturbations. Such perturbations can be, e.g., that the local gas density increases wherever the initial ambient magnetic field is less than average. We follow the standard analysis approach presented by Kato, Abramowicz \& Chen (1996). Taking the surface density as example, we denote perturbations $\Sigma_{1}$ superimposed over the unperturbed quantities $\Sigma$ as $\sigma \equiv \Sigma_{1} / \Sigma \propto \exp (n \Omega t-i k r)$ where $n \Omega$ is the growth rate of perturbation and $k$ is the perturbation wave number. Substituting the perturbed quantities to the time-dependent accretion equations, we can obtain the perturbed equations and the dispersion relation. The arguments for ADAFs presented in Kato, Abramowicz \& Chen (1996) hold for LHAFs as well although the advection is negative and radiative cooling is important here. We assume thermal conductivity is suppressed by the presence of the tangled magnetic field in the accretion flow (but see Narayan \& Medvedev 2001). As in the case of cooling flow, the presence of thermal conduction will strongly stabilize the flow. The dispersion relation is,

$$
-3 n \Omega \sigma=G \sigma
$$

and the condition for instability is

$$
G \equiv-\frac{Q_{\mathrm{vis}}}{W}\left(\frac{\partial \ln \eta}{\partial \ln \Sigma}\right)_{W}-\frac{Q_{\mathrm{rad}}}{W}\left(\frac{\partial \ln Q_{\mathrm{rad}}}{\partial \ln \Sigma}\right)_{W}<0 .
$$

Here $W \equiv 2 H p$ and $\eta \equiv \nu \Sigma$. Since $\eta \propto \Sigma c_{s}^{2} / \Omega \propto W / \Omega$, so $(\partial \ln \eta / \partial \ln \Sigma)_{W}=0$. For a one-temperature LHAF, $Q_{\mathrm{rad}} \propto H \rho^{2} T^{1 / 2} \propto \Sigma^{2} T^{1 / 2} / H \propto \Sigma^{2}$, so

$$
\left(\partial \ln Q_{\mathrm{rad}} / \partial \ln \Sigma\right)_{W}>0 .
$$

Therefore one-temperature LHAFs are thermally unstable against local perturbations.

The case of two-temperature LHAF is a little bit more complicated. Treating the energy equation in Kato et al. (1996) as the energy equation of ions, the instability condition is exactly the same as eq. (9) except that we should replace $Q_{\text {rad }}$ with $Q_{i e}$, the Coulomb collision cooling rate. Since $Q_{i e} \propto \rho^{2} H T_{e}^{-3 / 2} T_{i}$, to determine the sign of $G$, we must turn to 
the electron energy equation to obtain the relation between $T_{e}$ and $T_{i}$. Writing the energy equation of electrons as $Q_{\mathrm{adv}, \mathrm{e}}=Q_{i e}-Q_{\mathrm{rad}, \mathrm{e}}$, with $Q_{\mathrm{adv}, \mathrm{e}}, Q_{i e}$ and $Q_{\mathrm{rad}, \mathrm{e}}$ are the advection by electrons, Coulomb coupling, and radiative cooling of electrons respectively, the global solution of Paper I indicates that for LHAF a good approximation to the electrons energy equation is $Q_{i e} \approx Q_{\text {adv,e }}$ in the region outside of $\sim 100 R_{s}$ and $Q_{i e} \approx Q_{\text {rad,e }}$ inside $100 R_{s}$ (see Figure 5 in Paper I). Taking $Q_{i e} \approx Q_{\mathrm{adv}, \mathrm{e}}$ we can get $T_{e} \propto W^{1 / 5} \Sigma^{1 / 5}$. Hence $Q_{i e} \propto \Sigma^{6 / 5} W^{1 / 5}$ and

$$
G=-\frac{Q_{i e}}{W}\left(\frac{\partial \ln Q_{i e}}{\partial \ln \Sigma}\right)_{W}=\frac{6}{5}>0 .
$$

So a two-temperature LHAF is thermally unstable at least at the region outside of $\sim 100 R_{s}$. Inside $\sim 100 R_{s}$, the radiative cooling is in general dominated by the thermal Comptonization. The seed photons can be the synchrotron photons, or other soft photons from cold matter such as a SSD underlying or outside of the LHAF, or cold clumps suspending in the hot LHAF. The origin of the cold clumps in LHAFs can be due to the thermal instability of LHAFs (see below), or originally existed in the accretion material. For luminous sources the soft photons from the cold matter is likely to dominate over the synchrotron photons as the seed photons of Comptonization (Zdziarski et al. 1998; Wardzinski \& Zdziarski 2000). Since $Q_{\mathrm{rad}, \mathrm{e}}=\left(4 k T_{e} / m_{e} c^{2}\right) \rho H \kappa_{e s} U_{r} c$, with $U_{r}$ denotes the flux of seed photon, using $Q_{i e} \approx Q_{\mathrm{rad}, \mathrm{e}}$, we find that if $U_{r} \propto T_{i}^{2}$ or stepper, the two-temperature LHAFs will be thermally stable, otherwise it is unstable. Unfortunately, it is not easy to determine whether this condition can be satisfied or not, because of the complicated energy interaction between the cold and hot phases in accretion flow (e.g., Ferland \& Rees 1988).

Now let's discuss the consequence of the thermal instability against local perturbations. An important factor is how fast the perturbations grow compared to other related timescales. It is believed that as the result of instability cold dense clumps will form in the hot flow if the growth of perturbations is fast enough. This mechanism has been used to explain the condensations of galaxies from the intergalactic medium, formation of solar prominence, and condensations in planetary nebulae (e.g., Field 1965). In the case of accretion flows, we should compare the accretion timescale $t_{\text {acc }}$ with the growth timescale of perturbations $t_{\text {grow }}$. For LHAFs, from eq. (8), the timescale of the growth of local perturbations is

$$
t_{\text {grow }}=(n \Omega)^{-1}=3 G^{-1}=3\left[\frac{Q_{\mathrm{rad}}}{W}\left(\frac{\partial \ln Q_{\mathrm{rad}}}{\partial \ln \Sigma}\right)_{W}\right]^{-1}=\frac{3 W}{2 Q_{\mathrm{rad}}}
$$

This equals the thermal timescale of accretion flows, $t_{\mathrm{th}}$,

$$
t_{\text {grow }}=\frac{3(\gamma-1)}{2} t_{\mathrm{th}}=t_{\mathrm{th}}
$$

for adiabatic index $\gamma=5 / 3$. 
We compare the two timescales, $t_{\text {acc }}$ and $t_{\text {grow }}$, by numerical calculations. This needs us to solve the global solution of two-temperature LHAFs. We find that while the quantitative results depend on the values of parameters such as the viscous parameter $\alpha$, the qualitative results not. Two lines in Fig. 2 represent two kinds of typical results. The corresponding two models are identical to the two LHAF solutions presented in Figure 4 of Paper I, with the solid line corresponding to $\dot{m}=0.1\left(<\dot{m}_{2}\right.$, so the accretion flow is hot throughout the disk), and the dashed line to $\dot{m}=0.3\left(>\dot{m}_{2}\right.$, so the hot accretion flow collapses at a radius and form a cold annulus). We see that when the accretion rate is not very large, as shown by the solid line, $t_{\text {grow }} / t_{\text {acc }}>1$, so the perturbations have no time to grow before they are swallowed by the black hole. Therefore, the thermal instability will have no effect on the dynamics of LHAFs. When the accretion rate is very large, as shown by the dashed line, the growth of perturbation will be faster than accretion at the transition radius, if LHAFs are thermally unstable at that region. As a result of thermal instability, some cold dense clumps will form in hot flows. We speculate that once the condensation of the hot phase makes $t_{\text {grow }} \approx t_{\text {acc }}$, the condensation will stop. Quantitatively calculating the physical states of clumps such as their filling factor, density and temperature, is complicated and beyond the scope of the present Letter.

In our calculations of $t_{\text {acc }}$ and $t_{\text {grow }}$, we only take into account the "standard" radiative processes, namely synchrotron and bremsstrahlung emission and their Comptonization. If we include additional cooling, such as the Comptonization of additional soft photons from the cold phase, $t_{\text {grow }}\left(\equiv t_{\text {th }}\right)$ will decrease somewhat (and both $\dot{m}_{1}$ and $\dot{m}_{2}$ will also decrease). But we expect that the result that $t_{\text {grow }}>t_{\text {acc }}$ when $\dot{m}<\dot{m}_{2}$ will remain correct.

Note that LHAF will not collapse into an optically thick cold disk as the result of local perturbation since the perturbation wavelength is smaller than the disk scaleheight. The thermal stability of LHAFs under long wavelength perturbation is hard to determine, and it is also not clear if they are unstable how fast the growth of long scale perturbations is compared to the accretion timescale. On the other hand, if the filling factor of the cold clumps is large enough, it is possible that these clumps may assemble due to frequent collisions and form a disk-like large scale structure.

At last, we would like to note in this context that many authors have proposed such cold/hot two-phase accretion flow model for AGNs (e.g., Guilbert \& Rees 1988; Ferland \& Rees 1988; Celotti, Fabian, \& Rees 1992; Kuncic, Celleti \& Rees 1997). But almost all these work focus on the thermal state of cold clumps and how the clumps re-radiate the energy they absorb. SLE and Krolik (1998) also suggested a two-phase accretion flow model. But different from our suggested physical mechanism of clumps formation, they suggested that such a two-phase feature is the result of the instability in the radiation-pressure-dominated 
innermost region of a SSD (see e.g., Gammie 1998).

F.Y. is very grateful to Shoji Kato for many helpful discussions on the thermal stability of hot accretion flow. The discussions with Andrei Beloborodov, Ramesh Narayan are acknowledged. This work was supported in part by NASA grant NAG5-10780.

\section{REFERENCES}

Abramowicz, M.A., Chen, X., Kato, S., Lasota, J.-P., \& Regev, O. 1995, ApJ, 438, L37

Abramowicz, M.A., Czerny, B., Lasota, J.P., \& Szuszkiewicz, E., 1988, ApJ, 332, 646

Abramowicz, M.A., Chen, X.-M., Granath, M., Lasota, J.-P, 1996, ApJ, 471, 762

Begelman, M.C. \& Meier 1982, ApJ, 253, 873

Bjönsson G., Abramowicz M.A., Chen, X., \& Lasota J.-P. 1996, ApJ, 467, 99

Celotti, A., Fabian, A.C., \& Rees, M.J. 1992, MNRAS, 255, 419

Chen, X., Abramowicz, M.A., Lasota, J-P, Narayan, R. \& Yi, I. 1995, ApJ, 443, L61

Esin, A.A., McClintock, J. E., \& Narayan, R. 1997, ApJ, 489, 865

Fabian, A.C., \& Nulsen P.E.J. 1977, MNRAS, 180, 479

Ferland, G.J., \& Rees, M.J. 1988, ApJ, 332, 141

Field, G.B. 1965, ApJ, 142, 531

Frank, J., King, A., \& Raine, D. 1992, in Accretion Power in Astrophysics. Cambridge Univ. Press

Gammie, C.F., 1998, MNRAS, 297, 929

Guilbert, P.W., \& Rees, M.J. 1988, MNRAS, 233, 475

Ichimaru, S., 1977, ApJ, 214, 840

Kato, S., Abramowicz, M.A., \& Chen, X., 1996, PASJ, 48, 67

Kato, S. Fukue, J., \& Mineshige, S. 1998, Black-hole Accretion Disks (Kyoto University Press, Kyoto) 
Kim, W.T. \& Narayan, R. 2003, ApJ, submitted (astro-ph/0303097)

Krolik, J.H. 1998, ApJ, 498, L13

Kuncic, Z., Celotti, A., \& Rees, M.J. 1997, MNRAS, 284, 717

Kusunose, M. \& Mineshige, S. 1996, ApJ, 468, 330

Luo, C. \& Liang, E.P. 1994, MNRAS, 266, 386

Mathews, W.G., \& Bregman J.N., 1978, ApJ, 224, 308

Narayan, R., Mahadevan, R., \& Quataert, E. 1998, in "The Theory of Black Hole Accretion Discs", eds. M.A. Abramowicz, G. Björnsson, and J.E. Pringle (Cambridge University Press)

Narayan, R. \& Medvedev, M. 2001, ApJ, 562, L129

Narayan, R. \& Yi, I. 1994, ApJ, 428, L13

Narayan, R. \& Yi, I. 1995, ApJ, 444, 231

Nulsen, P.E.J. 1986, MNRAS, 221, 377

Paczyński, B., \& Wiita, P. J. 1980, A\&A, 88, 23

Pringle, J.E., 1976, MNRAS, 177, 65

Rees, M.J., Begelman, M.C., Blandford, R.D., \& Phinney, E.S., 1982, Nature, 295, 17

Shakura, N. I.\& Sunyayev, R. A. 1973, A\&A, 24, 337 (SSD)

Shapiro, S.L., Lightman, A.P., \& Eardley, D. M., 1976, ApJ, 203, 697 (SLE)

Yuan, F. 1999, ApJ, 521, L55

Yuan, F. 2001, MNRAS, 324, 119 (Paper I)

Zakamska, N.L. \& Narayan, R. 2003, ApJ, 582, 162

Zdziarski A. A., et al. 1998, MNRAS, 301, 435

Zdziarski A.A., 1998, MNRAS, 296, L51

This preprint was prepared with the AAS IATEX macros v5.0. 


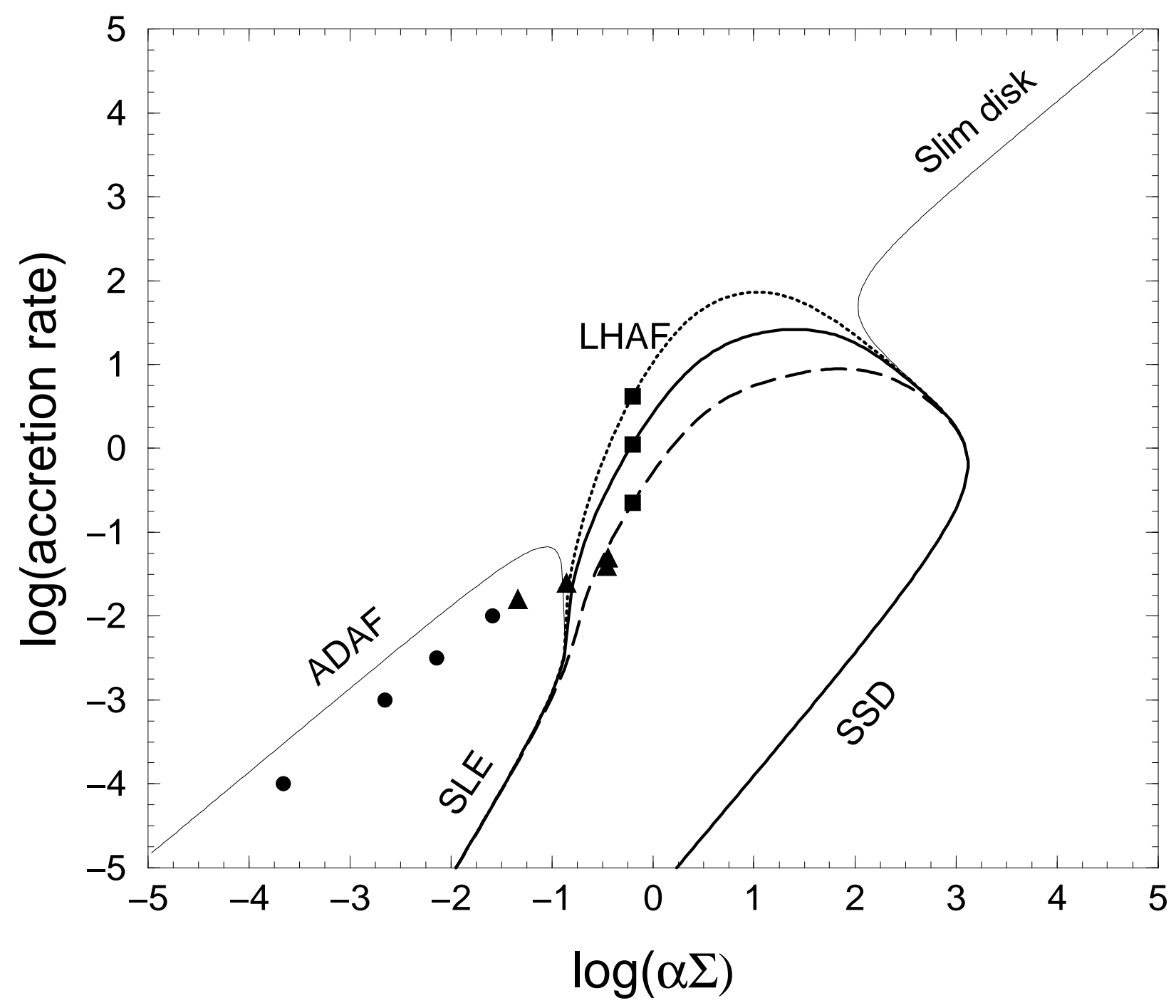

Fig. 1.- The thermal equilibrium curve of various accretion solutions. The accretion rate is in units of $\dot{M}_{\mathrm{Edd}} \equiv 10 L_{\mathrm{Edd}} / c^{2}$ and the units of $\Sigma$ is $\mathrm{g} \mathrm{cm}^{-2}$. The parameters are $M / M_{\odot}=$ $10, \alpha=0.1$, and $R=5 R_{s}$. The thin solid lines are for $\xi=1$, representing ADAF, SLE, SSD (the standard thin disk), and slim disk. The thick solid, dotted and dashed lines are for $\xi=-1,-0.1$ and -10 , respectively, representing LHAF solutions. The squares show the location of unity scattering optical depth. The filled circles and triangles denote the global solutions of ADAFs and LHAFs, respectively, for a one-temperature accretion flow. 


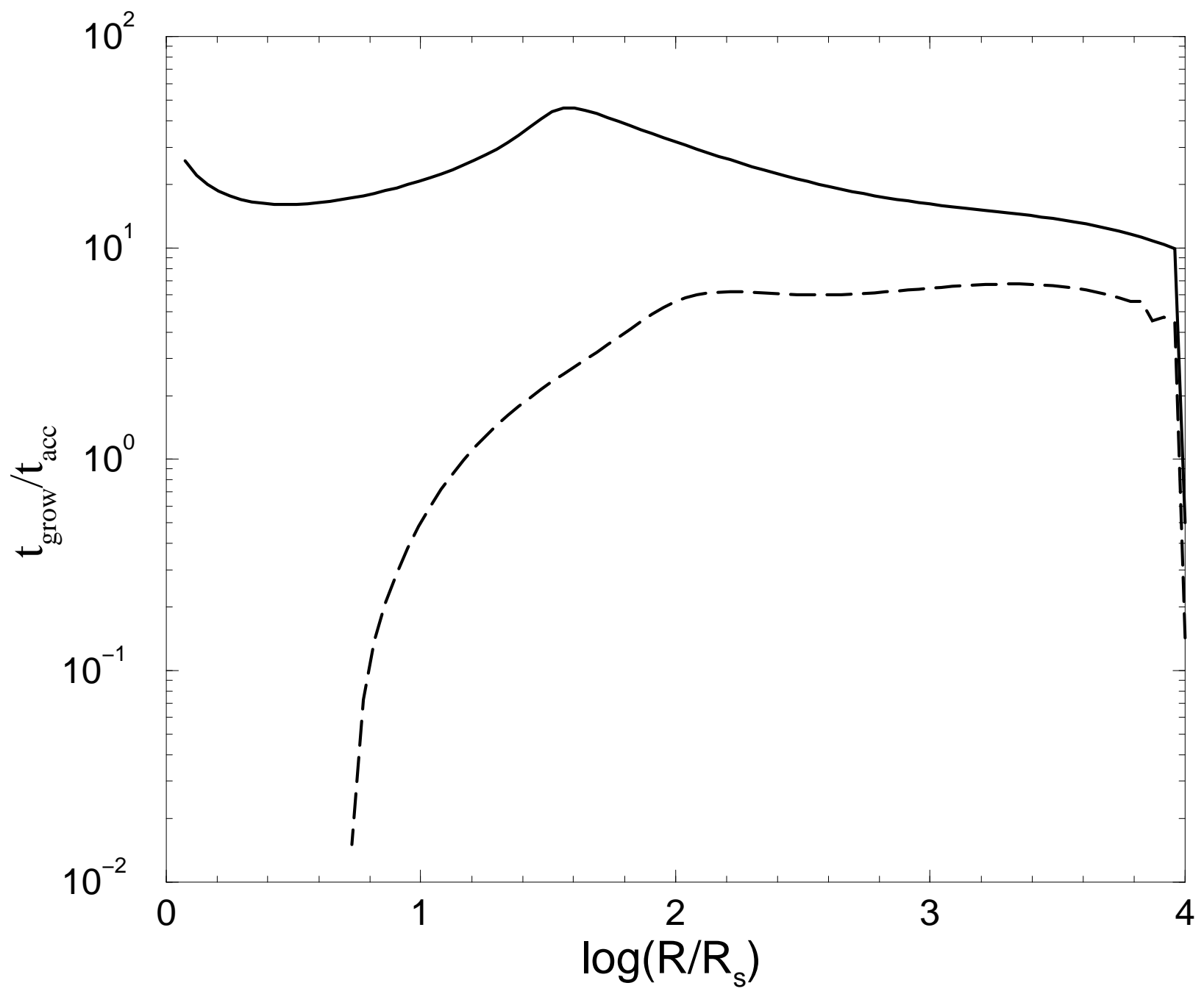

Fig. 2.- The ratio of the accretion timescale to the growth of perturbations timescale as a function of radii for two LHAF solutions presented in Fig. 4 of Paper I. The solid (dashed) line is for $\dot{m}=0.1(0.3)$. Other parameters are $\alpha=0.1, \beta=0.5$. 